\title{
Addendum et Corrigendum
}

\section{The Voyage of RAD: From the Old World to the New}

\section{by KENT M. HAWORTH*}

The metaphor of a voyage is appropriate for the title of this paper, symbolizing as it does the various characteristics of the descriptive standards development process in Canada. Voyages of discovery usually carry with them elements of challenge, new responsibilities, risk, and insecurity - not only for the crew but also for the sponsors of the enterprise. So it is with the development of Rules for Archival Description $(R A D)^{1}$ in Canada. Furthermore, any major voyage requires immense preparation, careful planning, and cooperation among the participants. Canada has benefited both from the important preparatory work undertaken by the authors of Toward Descriptive Standards (the report of the Canadian Working Group on Archival Descriptive Standards) and the financial commitment of archival institutions through the Canadian Council of Archives. ${ }^{2}$

The development of descriptive standards in Canada has also meant a voyage of rediscovery for many of those involved directly or indirectly in the process. It has meant revisiting and reexamining the theoretical foundations on which archival description rests and testing their applicability to archival description.

What distinguishes $R A D$, both in the way it has developed and in its content from other similar enterprises undertaken in Britain and the United States, is indicative of the Canadian archival culture. While there are some differences-discussed below-among $M A D, R A D$, and $A P P M$, there are also similarities. The differences should not deflect attention from the larger significance which these three enterprises represent as national efforts to standardize the way archivists describe archival materials. ${ }^{3}$ Canadians would like to think that, even though they may be a late entry in the descriptive standards enterprise, theirs is the best set of rules and that they have benefited from the mistakes of those who have gone before. However, when all is said and done, what each undertaking is trying to do nationally is to build a vessel commissioned for a common objective. Each has living quarters, galleys, bunks, corridors, and heads situated in different locations perhaps, with variations in some of the furnishings. There may be more rooms in some and less in others. The colour scheme may differ, but the galley stove is not likely to be found in the sleeping-quarters.

N.B. Kent Haworth's article, which was originally published in Archivaria 35 (Spring 1993), pp. 55-63, has had to be reprinted in its entirety because three full pages between those numbered 56 and 57 were omitted at the printing stage. Readers are therefore advised to disregard the incomplete former version of the article that was inadvertently published in Number 35 . 


\section{The Canadian Archival Culture}

Rule's for Archival Description is a microcosm of the nation which has produced it. It is a document developed through consensus and compromise and is a blend of French-Canadian and English-Canadian archival thought. The development of descriptive standards in Canada is distinguished particularly by the consultative process which the Planning Committee on Descriptive Standards (PCDS) established. Unlike Great Britain or the United States-where descriptive standards development has been largely the initiative and responsibility of one or two individuals - in Canada working groups are responsible for the presentation of a report to the PCDS-and subsequently to the profession-which includes a draft set of rules for a particular media chapter. The Canadian archival community has an opportunity to comment on each set of rules as they are drafted, and those which are published have been improved as a result of this consultative process.

$R A D$ also reflects the Canadian archival tradition, described as the "Total Archives" approach, where archival institutions, unlike European or United States archives, acquire both public or government archives and private (corporate and individual) archives in all documentary formats. ${ }^{4}$ Thus $R A D$ is comprehensive, containing rules for the description of a fonds and its parts, regardless of the form of the material created by individuals or corporate bodies acting in public or private capacities. Just as the Canadian archival tradition can be characterized as Total Archives, so RAD might be characterized as "Total Description"-enabling archivists to use $R A D$ independently, without reference to other cataloguing manuals, in order to describe "all forms of material ... uncommon material and material yet unknown" and to provide nonsubject access points for the purposes of retrieval. ${ }^{5}$

\section{The Foundation of Principle}

$R A D$ 's genesis can be found in the recommendations of Toward Descriptive Standards, the report of the Canadian Working Group on Archival Descriptive Standards. This report emphasized the importance of applying archival principles of arrangement to description, specifically the principle of respect des fonds. The Canadian preference for the French term fonds to define the records of one creator originated with Toward Descriptive Standards, which purposely chose it in order "to avoid certain terminological confusion which has grown around the terms 'record group,' 'manuscript group,' 'collection,' and so on, in North American practice." 6 Exactitude and rigour in defining the terms used in $R A D$ and ensuring the consistent application of principles to the drafting of the rules has been a major preoccupation of the PCDS and its working groups, and gives an essential integrity to $R A D$. This integrity reveals itself in the way $R A D$ has formulated its rules based on the archival principle of respect des fonds and the bibliographic structure of the ISBD(G). RAD embraces a respect for archival theory and presents a technique of multilevel description adapted from the bibliographic model for its application. Anchored as it is by the principle of respect des fonds, which governs the arrangement of archives, $R A D$ is applied only after arrangement has been completed. ${ }^{7}$ Accordingly, descriptions produced through $R A D$ should represent the structure of a fonds as it is informed by the principle of respect des fonds, and by means of the technique of multilevel description. In addition, by establishing the foundation for the structure of the rules on the principle of respect des fonds, it perforce establishes the premise that the formal process of description must take place after both the accessioning and the arrangement processes. RAD thus distinguishes between accessioning (the process of establishing physical and administrative control over archival materials) and description (the process of establishing intellectual control). ${ }^{8}$

There are those who prefer to consider only the first aspect of description (the theoretical underpinnings of descriptive practice). Others prefer to consider only the second (the application), and they can be characterized by their own plea: "Just give us the rules and we'll do it." 
What both groups fail to grasp is that the interplay of theory and practice is essential for the enrichment of both. ${ }^{9}$ It is akin to the novice sailor, who has no understanding of navigational principles, saying, "Give me the helm and I'll sail the ship," while the expert knowledgeable in the theory of navigation suffers from extreme motion sickness and never takes the voyage so as to witness the theory applied in practice.

\section{The Adoption of the Bibliographic Model}

While archival principle has been the foundation for the development of descriptive standards in Canada, the bibliographic model has been its structure. The Canadian rationale for choosing a bibliographic model was different from the American motivation. The genesis of Archives, Personal Papers, and Manuscripts (APPM) can be found in the Manuscript Division of the Library of Congress and the dissatisfaction of librarians there with the treatment of manuscripts in Chapter 4 of the second edition of the Anglo-American Cataloguing Rules (AACR2). $A P P M$ was originally drafted as a revision to $A A C R 2^{10}$ specifically to provide rules for the creation of bibliographic records; that is, the creation of archival descriptions derived from already existing finding aids for inputting into machine-readable catalogues, such as the $\mathrm{Li}$ brary of Congress, the Research Libraries Information Network (RLIN), and On-line Computer Library Center (OCLC) ${ }^{11}$ In this respect, $A P P M$ can be viewed more as a product of the historical manuscripts tradition than the public archives tradition in the United States. ${ }^{12}$

In Canada, the authors of Toward Descriptive Standards emphasized "the need to build on existing standards wherever possible and avoid reinventing the wheel." 13 They assessed the library model for bibliographic description and recommended its adoption in the absence of any comprehensive and systematic model of archival description covering all forms of archival material. Their recommendation was based on the premise that fundamental archival principles would not be compromised. They acknowledged that while some Canadian archivists wished to maintain staunch isolation from the library world, others recognized the wasted effort in starting from scratch. The first working group established by the PCDS to draft rules for a fonds d'archives at the fonds level endorsed the adoption of the bibliographic modelwith modifications- to ensure adherence to archival principles.

Adopting and adapting a bibliographic structure has meant leading archivists into unfamiliar territory where a residue of misunderstanding between the two related professions abounds. Unlike American archivists, whose field has been intertwined with librarianship, the relationship between librarians and archivists in Canada, as in Europe, has been an uneasy one. ${ }^{14} \mathrm{As}$ a result, Canadian archivists have warily approached the use of a bibliographic model to describe archival materials. Without doubt, there was no conscious effort to design a set of rules to produce descriptions that could readily be imported into bibliographic databases. It is not entirely accurate, therefore, to suggest that because $R A D$ follows a bibliographic model, the forms which the archival descriptions take are entries for a bibliographic database and thus "additional to the normal work of repositories." ${ }^{5}$ While $R A D$ adheres to a model that replicates a bibliographic structure, it does not necessarily follow from this that all $R A D$ descriptions will be bibliographic descriptions suitable only for bibliographic databases.

This is because $R A D$ is a data-content standard rather than a data-structure standard. $R A D$ 's focus is not on the products of description (e.g., various types of finding aids), but on accurately representing the arrangement of a fonds and its parts. $R A D$ leaves to institutions the responsibility for the design of output formats, which in turn are dependent on the type and purpose of whatever system of finding aids the institution chooses. ${ }^{16}$ This is one area where $R A D$ clearly distinguishes itself from its British and American cousins, most particularly from the Manual of Archival Description (MAD). MAD is intended to be a standard for the production of finding aids; it has rejected the bibliographic model as a standard for archival description. ${ }^{17}$ This position was recently endorsed by the Information Technology Standards working 
party of the National Council on Archives, when it "reject[ed] MARC, as a national standard for archival description in the United Kingdom." 18 MAD's focus on more rigorous models for output reflects perhaps the British archival tradition, where more emphasis has been placed on classification schemes and custodianship than on description. ${ }^{19}$

In addition, by rejecting the bibliographic model for description, $M A D$ is not required to address the matter of rules for the standardization of access points which make the retrieval of information both effective and efficient. The bibliographic model for description, on which $R A D$ - like $A P P M$ - is based, consists of two parts: a description of the material itself, and the provision of access points leading users to the identification of material relevant to their research. While on one hand, $M A D$ notes that the purpose of archival description is to create an effective "representation" of the original material, and recommends the indexing of descriptions for retrieval purposes, it gives no instructions on how to choose access points, what form they should take, and how they should be controlled. ${ }^{20}$ The PCDS, on the other hand, has taken a comprehensive view of archival description, and has commissioned two publications for archivists on authority control: a study of subject-indexing for archival materials and an examination of the concept of the fonds as it applies to description. ${ }^{21}$

\section{Charting a Careful Course}

The PCDS has endeavoured to ensure that $R A D$ adheres to archival principles, and at the same time maintain the integrated approach of the bibliographic model. From the outset, the drafting of the rules was afflicted with a tension between archival and bibliographic principles. This tension crystallized around the following contrasts: first, the bibliographic focus on the principles of the description of items (as opposed to groupings of archives, based on arrangement); secondly, the bibliographic emphasis of the description of the physical class of material in hand (and the archival tendency to highlight the intellectual); ${ }^{22}$ thirdly, the tension between the bibliographic concept of authorship and the archival concept of creatorship. With regard to the first, the PCDS had clear direction from Toward Descriptive Standards: any rules for archival description had to incorporate the concept of levels of description, from the highest (the fonds) to the lowest (the item).

As for the second, $R A D$ acknowledges the archivist's inclination to focus on the intellectual characteristics of the material being described, but believes that this descriptive tradition is not compromised by the adoption of the bibliographic model. To accommodate this preference, $R A D$ established the Archival Description Area, which contains the administrative history/ biographical sketch, custodial history, and the scope and content note. ${ }^{23}$

Finally, the third area of tension, that which persists between authorship and creatorship, manifests itself particularly in the differences between item-level description and description at higher levels. The creator of a fonds may be different from the individual responsible for the intellectual or artistic content of, for example, a letter or an audio-cassette. Recognizing this tension between the concept of authorship and creatorship, $R A D$ has, for example, distinguished between the formation of a title at higher levels and the formation of a title at lower levels. ${ }^{24}$

$R A D$ has endeavoured as much as possible to adhere to the integrity of the ISBD structure when it can do so without compromising archival principles. APPM alters the ISBD structure by, for example, moving the date field into the title area. ${ }^{25}$ The bibliographic tradition requires that all information not taken from the chief source - that is, supplied information-be placed in square brackets. $A P P M$ and $R A D$ both eliminate square brackets from supplied information for good reason: most information at higher levels of description for archival materials is supplied information. Nevertheless, $R A D$ still requires that the source of supplied information be provided in a note. ${ }^{26} A P P M$, unlike $R A D$, gives precedence to finding aids over the archival materials themselves as the chief source. ${ }^{27}$ This decision is derived from the original objective 
behind APPM: to create catalogue records of archival finding aids rather than representations of the archival materials themselves. In the treatment of reproductions, APPM appears to equivocate by leaving unanswered the question of what dates to use when describing original material on microform. ${ }^{28}$ The differences noted here are subtle and reflect perhaps a more pragmatic approach, indicative of the American cultural imperative. ${ }^{29}$

$R A D$ 's voyage on a sea of archival principle in a bibliographic vessel inevitably led it to an examination of the technique of multilevel description. The PCDS considered the technique of multilevel description (expressed only briefly in Rule 13.6 of $A A C R 2 R$ ) to be integral to the accurate representation of archival material and accordingly elevated it prominently to a separate chapter. $R A D$, like $M A D$, places special emphasis on multilevel description. As Michael Cook has pointed out, archival descriptions are representations of structural divisions within a complex, dynamic entity; namely, the fonds d'archives of a person, family, or corporate body naturally created and/or accumulated and used in the conduct of that creator's activities or functions. ${ }^{30}$ In $A P P M$, levels of description are based on "provenance or physical form." ${ }^{31}$ In $R A D$ and $M A D$, they are based on arrangement.

The similarities and differences highlighted above among the approaches of RAD, MAD and $A P P M$ to standardizing archival description reflect different approaches behind the development of descriptive standards for archives in the three countries. Those differing intentions reflect the archival cultures from which they have emanated, and a sensitivity for the extent to which the standards they propose will be accepted by the archival community which must apply them. What are some of the effects on the Canadian archival culture of implementing $R A D$ ?

\section{Voyaging to the New World}

The appearance of $M A D, R A D$, and $A P P M$ signals a turning-point for archivists in their approach not only to description, but also to their relationship with users and related professions, most notably librarians, documentalists, and other information management professionals. In the course of considering the development of descriptive standards, archivists are beginning to look outward, rather than inward. This is in part a consequence of the impact of automated systems. Information exchange networks present exciting and challenging new opportunities for archivists. The communication networks that are being developed locally, nationally, and internationally have the potential to provide us with the means to communicate information about our holdings to a larger audience. The more Canadian archivists begin to use RAD and discover that it structures, rather than changes, the way they have traditionally described their holdings, the more comfortable they will become with its use.

In addition, $R A D$ has given archivists a formal set of stated requirements for the description of information that has archival value. This has significant implications for the management of information across the life cycle of the information management process. Archival records are created long before they arrive in archival repositories. With a standardized table of data elements, archivists could identify those elements which might be incorporated into a records retention schedule because the information contained in the data element remains stable across the life cycle of the record (e.g., series titles, restrictions on access, accrual notes, administrative histories). Consequently, the data collection activity necessary for accurate descriptions of information that takes place at various points across the life cycle continuum could be both standardized and reduced. As a set of rules designed to standardize the communication of information about information, a data content standard such as $R A D$ has the potential to influence the design of information management systems. As a result, it gives archivists an opportunity, if they care to exploit it, to have substantive input into the design of such systems, in other words "to have something to say" at the decision-making table. ${ }^{32}$ 
These are some of the positive effects which $R A D$ can have for archivists currently on the periphery of information technology. As with any voyage of discovery, there are dangers as well as potential benefits. Inasmuch as $R A D$ is a set of formal rules designed to standardize a particular archival function, it can be characterized as a technique; as Neil Postman points out, "... there is nothing to fear from techniques, unless, like so much of our machinery, they become autonomous." ${ }^{33}$ RAD, like MAD and $A P P M$, must be seen as a means rather than as an end. Postman warns us of the danger when "we tend to believe that only through the autonomy of techniques (and machinery) can we achieve our goals." The questions which Postman asks of techniques are ones which the archival profession and archival institutions must pose of $R A D$ : "Who is to be the master? Shall we control it, or will it control us?" ${ }^{34}$ In order for the profession to maintain mastery over $R A D$, archivists will have to pay careful attention to its implementation and ongoing maintenance. It will also require careful monitoring of the sensitive interplay between descriptive theory and descriptive practice as changes take place in the way information is created and transmitted. It means that the profession will have to invest in education and training programmes in order to facilitate the implementation of $R A D$. However much $R A D$ professes to be "output neutral," as noted above, it is indisputable that a description produced through $R A D$ can be accepted as a MARC record. Currently, three major information-processing monopolies in North America, UTLAS, RLG, and OCLC, are attempting to capture the archival market. Canadian archivists-especially the heads of archival institutionswill have to address the question of whom we want to administer the outputs and to consider the potential dangers of relinquishing a portion of our control over the management of our descriptive tools.

Descriptive standards have the potential to transport archivists from the old world to the new world of information management. The voyage is a costly one, requiring diligence, commitment, and patience for those who choose to embark on the journey. Nevertheless, the benefits for users, keepers, and sponsors of archives are undeniable and will, in the end, make the journey a rewarding one.

\section{Notes}

* Paper presented at the 1992 Annual Conference of the Association of Canadian Archivists, Montréal, 13 September 1992; revised and edited for publication.

1 Bureau of Canadian Archivists (Ottawa, 1992). References in this paper to MAD refer to Michael Cook and Margaret Procter, Manual of Archival Description, 2nd ed. (Brookfield, VT, 1989). APPM refers to Steven L. Hensen, Archives, Personal Papers, and Manuscripts: A Cataloguing Manual for Archival Repositories, Historical Societies and Manuscript Libraries, 2nd ed. (Chicago, 1989).

2 The Canadian Working Group recognized from the outset that it "could not hope to develop detailed standards for all media and would instead be preoccupied with the task of trying to plot a course rather than sail a ship": Toward Descriptive Standards (Ottawa, 1985), p. 4. The CCA ranked descriptive standards second among ten long-term planning priorities for the development of the archival system in Canada: Canadian Council of Archives, The Canadian Archival System: A Report on the National Needs Priorities of Archives, Summary Report-August 1989 (Ottawa, 1989), p. 25.

3 Indeed, it is in large measure because of the influence of these national initiatives that the International Council on Archives has established an Ad Hoc Commission on Archival Descriptive Standards, and drafted a "Statement of Principles Regarding Archival Description" (Ottawa, February 1992), and "General International Standard Archival Description [ISAD(G)] (Ottawa, January 1992).

4 The concept is articulated as an "attempt to document all aspects of historical development, seeking the records not just of officialdom or of a governing élite but of all segments of a community," and "combining official administrative records and related private files, architectural drawings, maps, microfilm, and other documentary forms all touching on the development of the organization or region ...": Canadian Archives, Report to the Social Sciences and Humanities Research Council of Canada (Ottawa, 1980), pp. 63-64.

$5 R A D$, (Rule 0.1.), APPM (Rule 1.0A), gives the archivist the alternative of consulting other rules, depending on the media, for medium-specific details, or for the entire description. Similarly, MAD "does not try to cater for the needs of repositories or archives services which are highly specialized, either because of the physical form of their materials (audio or machine-readable archives, for example) or because of their attachment to specialized agencies": MAD, p. xii. 
6 Toward Descriptive Standards, p. 7. APPM (Rule 1.0A) does not ameliorate this terminological confusion in its definitions of collections, record groups, and archives.

$7 \quad R A D$, Rules 0.1 and 0.2 .

$8 R A D$, Rule 0.2 .

9 Berner makes the following observation of librarians and archivists in the United States with respect to theory and practice: "Both [librarianship and archives], being heavily practical in emphasis, have tended to be wary of theory, as though practices would only be confounded by interaction with theory": Richard C. Berner, Archival Theory and Practice ... in the United States (Seattle, 1983), p. 5.

10 Michael Gorman and Paul W. Winkler, eds., Anglo-American Cataloguing Rules, 2nd ed. rev. (Ottawa, 1988).

11 APPM, Preface to the 2 nd ed., p. v.

12 For an exposition of the two traditions, see Berner, Archival Theory and Practice ... in the United States. APPM (Rule 0.2) is intended to replace Chapter 4 of AACR2, "Manuscripts in Textual Form," and directs users to other cataloguing manuals for the description of non-textual materials at the item level (Rules 0.3 and $1.0 \mathrm{~A}$ ). Even though $A P P M$ claims to cover the description of archival materials at higher levels (collection, series), regardless of physical form or medium, the form terms which it provides as examples (e.g., "papers," "records," "collection") do not appear to support the claim.

13 Toward Descriptive Standards, p. 77.

14 Berner, Archival Theory and Practice ... in the United States, p. 5. For a European view, see, for example, Elio Lodolini, "The War of Independence of Archivists," Archivaria 28 (Summer 1989), p. 41.

15 Michael Cook, "Standards for Archival Description and for Data Exchange," Janus (1992), p. 153.

16 RAD, Rule 0.2 .

17 MAD, pp. xii-xiii; Janus (1992), p. 153.

18 "Information Technology Standards and Archival Description," Report of a Working Party to the National Council on Archives (March 1991), p. 1.

19 As Michael Cook notes, even Jenkinson, "their principal writer and theorist ... had little interest in description": Michael Cook, "Standardization of Archival Description," Journal of the Society of Archivists 8, no. 3 (April 1987), p. 181.

$20 M A D$ (Rule 9.5G) states, "In general, the question of appropriate access points should be considered when finding aid systems are designed. Planning decisions will have their effect on the lists and indexes produced." MAD's information retrieval rule (Rule $8.4 \mathrm{~B}$ ) recommends that "descriptions should contain in their text all the keywords needed in order to provide retrieval in the circumstances envisaged for this representation," and acknowledges that "the range and accuracy of searches possible may be improved if an index vocabulary or thesaurus has been provided" (Rule 8.4B2). The lack of guidance for archivists with respect to the provision of access points is one of MAD's significant weaknesses.

21 Louise Gagnon-Arguin, An Introduction to Authority Control for Archivists (Ottawa, 1989); Elizabeth Black, Authority Control for Archivists (Ottawa, 1991); Subject Indexing for Archivists (Ottawa, 1992); and The Concept of the Archival Fonds (Ottawa, 1992).

22 Compare Rule 0.24 of $A A C R 2 R$ ("It is a cardinal principle of the use of Part I that the description of a physical item should be based in the first instance on the chapter dealing with the class of materials to which that item belongs") with $R A D$ rule 0.24 , which takes the same approach: "... the starting point for description is the physical class of material into which the descriptive unit falls."

23 See note 8 to Rule 0.24 in RAD. APPM, on the other hand, shifts "undue emphasis away from the physical aspects and more toward content-related elements": Hensen, "Squaring the Circle, The Reformation of Archival Description in AACR2," Library Trends (Winter 1988), p. 549.

$24 R A D$ has limited the use of a statement of responsibility as a statement of authorship intended for published items. Thus, it can be employed only when it appears in conjunction with a formal title proper in or on the chief source of information (Rule 1.1F1). As a result, statements of responsibility will seldom-if ever-appear at the fonds or series level.

$25 A P P M$ (Rule 0.10): “... a collection's inclusive dates are considered an integral part of its title." $R A D$ retains date information in the ISBD $(\mathrm{G})$ area-Area 4 : Publication, Distribution, etc., Area-prescribed for date information, but renames it "Dates of Creation, including Distribution, Publication, etc., Area." Ironically, the intervening "Edition Area" and "Material Specific Details Area" drop away in archival description, thereby ohviating the need for a departure from the $\operatorname{ISBD}(G)$ structure along the lines prescribed by $A P P M$. Hensen justifies the decision to place date information in the title and not in Area 4 on the grounds that, because "... manuscripts per se do not have publication dates, it was obviously inappropriate to use this area": Steven L. Hensen, "Squaring the Circle," p. 548. Compare the description of a literary manuscript following APPM with one following RAD: 
$R A D:$ My voyage of discovery, 1992. 2nd draft.

My voyage of discovery. 2nd draft. 1992.

The example demonstrates that retaining the ISBD structure, as $R A D$ does, results in less ambiguity, leaving no doubt in the user's mind about the date of creation of the second draft.

$26 A P P M$ (Rule 0.10): "Supplied titles are not enclosed in square brackets. $R A D$ (Rule 1.1B4) requires that when information in the title area is supplied, the source of the supplied title must be given in a note.

$27 A P P M$, Rules 0.10 and $1.0 \mathrm{~B} 1$, which place last in the hierarchy of sources the archival materials themselves. In $R A D$ (Rule 3,0B 1 and Rule 4.0B 1 ), the chief source of information for textual records and graphic materials at the fonds level is all the material in the fonds being described.

$28 A P P M$ provides for the recording of two physical extent statements when institutions hold both the original and a copy of the original, but gives no guidance on which dates to record: the dates of the original or the dates of the copy. $R A D$ 's attempt at a solution (Rule 1.IE7) to the complex problem of which dates to use when describing originals on microform, is to supply dates of the originals as other title information "if, when taken together, the title proper and date of creation for the reproduction is misleading." It is interesting to note here that the second edition of APPM does not contain the direction found in the first edition to "describe microform materials primarily in terms of the physical extent of the original material on the microform.... Information about the microform is added in parentheses": Steven L. Hensen, Archives, Personal Papers, and Manuscripts: A Cataloging Manual for Archival Repositories, Historical Societies, and Manuscript Libraries (Washington, DC, 1983), Rule 4.5B3, p. 18. This is an example of $A P P M$ reflecting the practice of the Library of Congress, whose interpretation of $A A C R 2$ rules relating to reproductions contradicts $A A C R 2$ by relegating to notes the description of the reproduction rather than of the original, as $A A C R 2$ instructs. For the effect of this distortion by the Library of Congress, see William E. Studwell, "The Library of Congress's Policy on Cataloging Microform Reproductions and Photocopies: A Half Way Measure," Cataloguing \& Classification Quarterly 14, no. 1 (1991), pp. 69-74.

29 Hensen, "Squaring the Circle," p. 542; APPM, Rule 0.2. Berner has observed that because the American "profession's approach has been preeminently practical, eclecticism has been rampant": Berner, Archival Theory, p. 5.

30 Michael Cook, The Management of Information from Archives (Aldershot, 1986), p. 104.

31 APPM, Rule 0.12. See also Wendy Duff and Kent Haworth, "The Reclamation of Archival Description: The Canadian Perspective," Archivaria 31 (Winter 1990-91), p. 32, and the exchange between Hensen and Heather MacNeil in the same issue of Archivaria, pp. 5-9.

32 Lisa Weber, "The Working Meeting on Research Issues in Electronic Records, A Report to SAA," 28 September 1991, p. 1.

33 Neil Postman, Technopoly: The Surrender of Culture to Technology (New York, 1992), p. 142

34 Ibid., p. 142. 